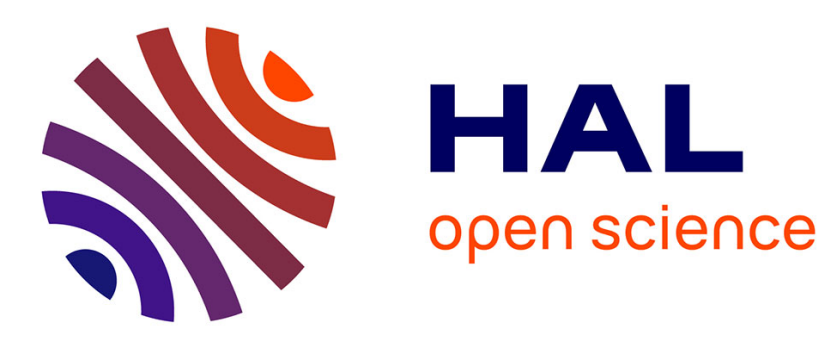

\title{
Stability of the triangular Lagrange points beyond Gascheau's value
}

B. Sicardy

\section{To cite this version:}

B. Sicardy. Stability of the triangular Lagrange points beyond Gascheau's value. Celestial Mechanics and Dynamical Astronomy, 2010, 107 (1-2), pp.145-155. 10.1007/s10569-010-9259-5 . hal-00552502

\section{HAL Id: hal-00552502 \\ https://hal.science/hal-00552502}

Submitted on 6 Jan 2011

HAL is a multi-disciplinary open access archive for the deposit and dissemination of scientific research documents, whether they are published or not. The documents may come from teaching and research institutions in France or abroad, or from public or private research centers.
L'archive ouverte pluridisciplinaire HAL, est destinée au dépôt et à la diffusion de documents scientifiques de niveau recherche, publiés ou non, émanant des établissements d'enseignement et de recherche français ou étrangers, des laboratoires publics ou privés. 


\title{
Stability of the triangular Lagrange points beyond Gascheau's value
}

\section{B. Sicardy}

Received: 2 November 2009 / Revised: 24 January 2010 / Accepted: 8 February 2010 /

Published online: 16 March 2010

(C) Springer Science+Business Media B.V. 2010

\begin{abstract}
We examine the stability of the triangular Lagrange points $L_{4}$ and $L_{5}$ for secondary masses larger than the Gascheau's value $\mu_{\mathrm{G}}=(1-\sqrt{23 / 27} / 2)=0.0385208 \ldots$ (also known as the Routh value) in the restricted, planar circular three-body problem. Above that limit the triangular Lagrange points are linearly unstable. Here we show that between $\mu_{G}$ and $\mu \approx 0.039$, the $L_{4}$ and $L_{5}$ points are globally stable in the sense that a particle released at those points at zero velocity (in the corotating frame) remains in the vicinity of those points for an indefinite time. We also show that there exists a family of stable periodic orbits surrounding $L_{4}$ or $L_{5}$ for $\mu \geq \mu_{G}$. We show that $\mu_{G}$ is actually the first value of a series $\mu_{0}\left(=\mu_{G}\right), \mu_{1}, \ldots, \mu_{i}, \ldots$ corresponding to successive period doublings of the orbits, which exhibit $1,2, \ldots, 2^{i}, \ldots$ cycles around $L_{4}$ or $L_{5}$. Those orbits follow a Feigenbaum cascade leading to disappearance into chaos at a value $\mu_{\infty}=0.0463004 \ldots$ which generalizes Gascheau's work.
\end{abstract}

Keywords Three-body problem · Lagrange points · Global stability · Routh's critical mass ratio · Feigenbaum cascade

\section{Introduction}

Euler and Lagrange were both awarded the Prix de l'Académie Royale des Sciences de Paris in 1772 for their work on the three-body problem. Euler was the discoverer of the collinear equilibrium points, now known as $L_{1}, L_{2}$ and $L_{3}$ (Euler 1763, 1764, 1765), while Lagrange had a more general approach, revealing also the triangular (equilateral) equilibrium points $L_{4}$ and $L_{5}$ (Lagrange 1873). 
Gascheau $^{1}$ was the first to study the stability of the $L_{4}$ and $L_{5}$ points (Gascheau 1843), showing that the motion of three non-zero masses $m_{1}, m_{2}$ and $m_{3}$ in a rotating equilateral configuration becomes linearly unstable if

$$
\frac{\left(m_{1}+m_{2}+m_{3}\right)^{2}}{m_{1} \cdot m_{2}+m_{1} \cdot m_{3}+m_{2} \cdot m_{3}} \leq 27 .
$$

This result was published more than thirty years earlier than Routh (1875), see also Routh (1905). Routh actually extended the result to a general attraction law $\propto 1 / r^{\kappa}$. He showed that the triangular points are linearly unstable for all masses if $\kappa$ is larger than 3 , and linearly stable for all masses if $\kappa$ is smaller than -1 . For $\kappa$ between -1 and 3, the triangular points are linearly unstable for:

$$
\frac{\left(m_{1}+m_{2}+m_{3}\right)^{2}}{m_{1} \cdot m_{2}+m_{1} \cdot m_{3}+m_{2} \cdot m_{3}} \leq 3\left(\frac{1+\kappa}{3-\kappa}\right)^{2},
$$

which is identical to Gascheau's expression for the gravitational case, $\kappa=2$. Note that further studies on the stabiltiy of the equilateral configuration as a function of $\kappa$ can be found in Santoprete (2006).

The value of $\mu_{G}=m_{2} /\left(m_{1}+m_{2}\right)=(1-\sqrt{23 / 27}) / 2=0.03852 \ldots$ at which linear unstability is reached in the restricted case $\left(m_{3}=0\right.$, see see Eq. 4$)$ is often referred to as the Routh value, but should actually be called Gascheau's value in view of the precedence of his result.

The linear stability of the triangular Lagrange points was subsequently studied by Danby (1964) and Bennett (1965) in the elliptic restricted case. These authors show, among others, that the maximum possible value of $\mu$ allowing a linearly stable motion near $L_{4}$ or $L_{5}$ is $\mu=0.04698 \ldots$, reached for an orbital eccentricity of $e=0.3143 \ldots$ of the primary and secondary. Above $\mu=0.04643 \ldots$ the triangular points are always linearly unstable, whatever the orbital eccentricities may be.

Further studies have gone beyond the first order analysis to investigate whether all values of $\mu$ between 0 and $\mu_{G}=0.03852 \ldots$ provide linear stability for $L_{4}$ or $L_{5}$. It was shown in Deprit and Deprit-Bartholomé $(1967)$ that for $\mu_{3: 1}=(15-\sqrt{233}) / 90=0.01352 \ldots$ and $\mu_{2: 1}=(45-\sqrt{1833}) / 90=0.02429 \ldots$, the triangular points are linearly unstable. These values actually correspond to the 3:1 and 2:1 resonances between the two eigenfrequencies of motion near $L_{4}$ or $L_{5}$, see the review by Marchal (1990). In the same review, it is also mentioned that $\mu_{3: 1}$ and $\mu_{2: 1}$ are actually the only values between 0 and $\mu_{G}$ yielding linearly unstable motion around the triangular points.

Note that the stability of motions around $L_{4}$ has been extensively considered for the special case of the Trojan asteroids, for which Jupiter's mass is fixed at about 0.001 of the solar mass. In that case, one may for instance want to estimate the stability of Trojans asteroids initially placed farther and farther away from the $L_{4}$, see e.g. Sándor et al. (2000) and Efthymiopoulos (2005). This problem will not be tackled here.

Rather, we study here the behavior of a massless particle near the triangular equilibrium points $L_{4}$ and $L_{5}$ for a secondary masse just above Gascheau's value in the simplest possible case, namely the restricted, circular and planar problem.

Notations are standard, $1-\mu$ being the mass of the more massive body (the primary), $\mu$ being the mass of the less massive body (the secondary), with $\mu \leq 0.5$. The case $0.5 \leq \mu \leq 1$

1 Gabriel Gascheau was born in 1798, he was a professor at Toulouse University, France. His thesis on "Mouvements relatifs d'un système de corps. Sur deux cas particuliers d'un problème des trois corps" was published in Paris in 1843. 
can be studied by a trivial exchange of the primary and secondary. The secondary moves on a circular orbit of radius $r_{s}=1$ and position vector $\mathbf{r}_{\mathbf{s}}$ relative to the primary, with mean motion $n_{s}=1$ and period $T_{s}=2 \pi$. Finally, a test particle moves with position vector and distance $\mathbf{r}$ and $r$, respectively, relative to the primary. Here we call "corotating frame" the frame centered at the primary and rotating at the secondary mean motion $n_{s}=1$. The particle motion will be studied in this frame, so that "motionless" will mean that the particle has zero velocity in the corotating frame.

The linear stability of points $L_{4}$ and $L_{5}$ is treated in many textbooks, see e.g. Murray and Dermott (1999). The proper frequencies $\lambda$ in the vicinity of the triangular points are solutions of:

$$
\lambda^{4}+\lambda^{2}+\frac{27}{4} \mu \cdot(1-\mu)=0
$$

For $\mu \rightarrow 0$, solutions are purely imaginary, $\lambda_{1} \approx \sqrt{27 / 4 \mu} \cdot j$ and $\lambda_{2} \approx j$, and their opposite values, with $j=\sqrt{-1}$. The frequency $\lambda_{1}$ correspond to stable slow oscillations of the particle around $L_{4}$ or $L_{5}$, along the so called "tadpole" orbits, while $\lambda_{2}$ correspond to the fast stable epicyclic motion with period $2 \pi$. As $\mu$ increases, $\left|\lambda_{1}\right|$ increases and $\left|\lambda_{2}\right|$ decreases, until $27 \mu \cdot(1-\mu)=1$, at which point the two frequencies merge together at $\left|\lambda_{1}\right|=\left|\lambda_{2}\right|=1 / \sqrt{2}$. When

$$
1 \leq 27 \mu \cdot(1-\mu)
$$

the solutions of Eq. 2 have non-zero real parts, so that the triangular points become linearly unstable. Note that Eq. 3 is identical to Eq. 1 once we take $m_{3}=0, \mu=m_{2} /\left(m_{1}+m_{2}\right)$ and $m_{1}+m_{2}=1$.

Gascheau's value is the smaller solution of $27 \mu \cdot(1-\mu)=1$ :

$$
\mu_{\mathrm{G}}=\frac{1-\sqrt{23 / 27}}{2}=0.0385208965 \ldots
$$

The other solution, $1-\mu_{G}$, is obtained by permutating the primary and the secondary.

Our main scope in this paper is to present an as simple as possible account of a particle's behavior near $L_{4}$ and $L_{5}$ for values of $\mu$ just above $\mu_{G}$. We actually want to answer the two following questions:

- Let us consider a particle initially motionless at $L_{4}$ or $L_{5}$. What is the global stability of the particle near those points for $\mu \geq \mu_{G}$ ? Namely, can the particle remain in a bounded region encircling $L_{4}$ or $L_{5}$ for some values of $\mu \geq \mu_{G}$, even if $L_{4}$ and $L_{5}$ are linearly unstable?

- Are there stable periodic orbits encircling the triangular points for $\mu \geq \mu_{G}$ ?

These questions may have some pertinence when studying the stability of a spacecraft motion near one of the triangular Lagrange points of a binary system with $\mu \geq \mu_{G}$. It may also be relevant to systems where particles are trapped near the $L_{4}$ or $L_{5}$ points of a system where the secondary undergoes a progressive mass increase, which eventually leads to $\mu \geq \mu_{G}$.

To tackle those problems, we perform numerical simulations using Poincaré surfaces of section, which help clarifying the structure of the web of orbits in the vicinity of $L_{4}$ or $L_{5}$, as $\mu$ goes beyond Gascheau's value. From the symmetry of the $L_{4}$ and $L_{5}$ points, we will only consider motions near $L_{4}$, unless otherwise stated. All our conclusions will however be applicable to the $L_{5}$ point, even if only $L_{4}$ is quoted in the text for sake of brevity. 


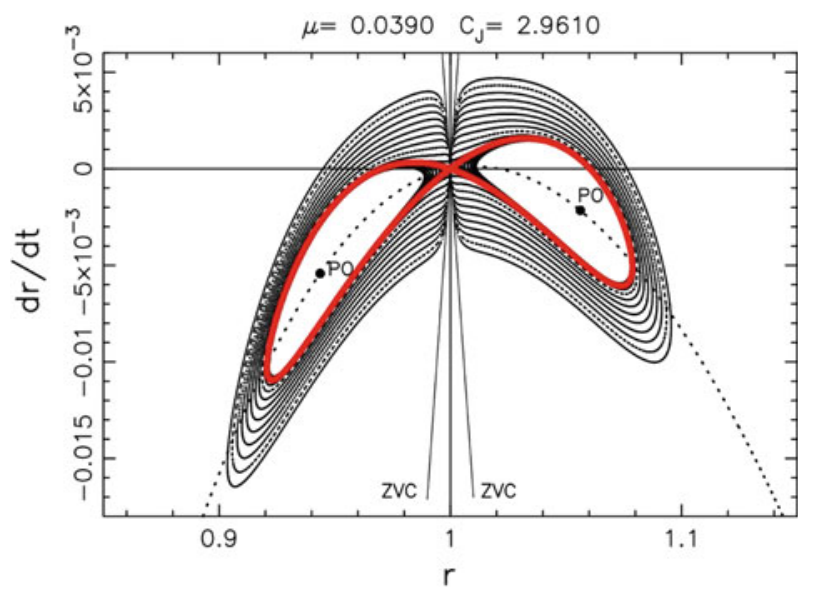

Fig. 1 Poincaré surface of section for orbits with $\mu=0.039$ and $C_{J}=2.961$, see text for details. The $t w o$ thin lines labelled "ZVC" are the zero-velocity curves, between which no motion is possible. The thick red curve is the orbit going through $L_{4}$, at $(r, \dot{r})=(1,0)$, with zero velocity. It corresponds to the orbit labelled (a) in Fig. 5. The two points labelled "PO" are the fixed points corresponding to the periodic orbit encircling $L_{4}$ for $\mu=0.039$, and shown in Fig. 6 (upper left orbit). The dotted curves are the locus of the fixed points when $\mu$ is increased slowly beyond Gascheau's value, see also Fig. 7 (colour version is available in the online version)

\section{Numerical integrations}

The restricted circular three-body problem has an energy-like integral of motion, the Jacobi constant:

$$
C_{J}=r^{2}+\frac{2(1-\mu)}{r}-2 \mu\left(\mathbf{r} \cdot \mathbf{r}_{\mathbf{s}}-\frac{1}{\left|\mathbf{r}-\mathbf{r}_{\mathbf{s}}\right|}\right)-v^{2}
$$

where $v$ is the particle velocity measured in a frame fixed at the primary center and corotating with the secondary. Here, we will first restrict ourselves to the case where the particle is initially motionless at $L_{4}(v=0)$. Those orbits are thus uniquely parametrized by the Jacobi constant, $C_{J}=3-\mu$.

Since the particle planar motion has two degrees of freedom and one integral of motion, we can readily construct Poincaré surfaces of section of its motion. As we study orbits encircling the triangular Lagrange points, we will plot the two conjugate quantities $r$ and $\dot{r}=d r / d t$ everytime the particle crosses the line joining the primary and the $L_{4}$ point. Note that if $\Phi=L-L_{s}$ denotes the difference between the true longitudes of the particle and the secondary, then the surface of section corresponds to $\Phi=\pi / 3$. A given orbit will in general intersect the surface $\Phi=\pi / 3$ twice, once with $\dot{\Phi} \geq 0$, and once with $\dot{\Phi} \leq 0$. We will not restrict the plotting of $(r, \dot{r})$ to one of those two cases, so that the surfaces of section will have a roughly symmetrical aspect (see e.g. Fig. 1), with both sides of the diagram containing the same information.

Orbits are calculated using a 4th order Runge-Kutta integrator with time step $h=0.01$. Intersections with the surface of section are calculated through a classical Newton's method. Conservation of energy is ensured at typical levels of $\left|\Delta C_{J} / C_{J}\right| \approx 2 \times 10^{-7}$ over typical times of $t \approx 10^{5}$, at which stage $\approx 10^{4}$ intersections with the surface of section are calculated. 

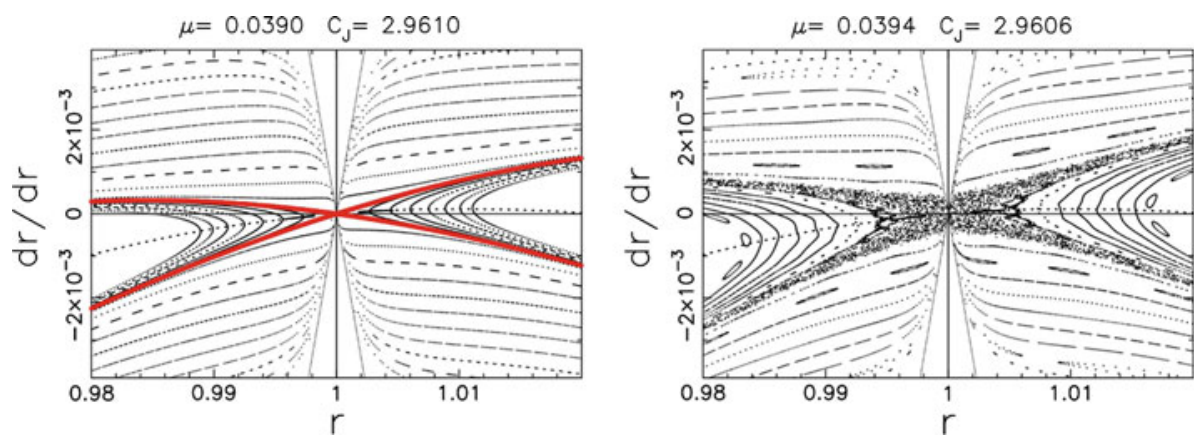

Fig. 2 Left panel An enlargement of the central region of Fig. $1(\mu=0.039)$. Right panel Same as left panel, but with $\mu=0.0394$ (colour version is available in the online version)
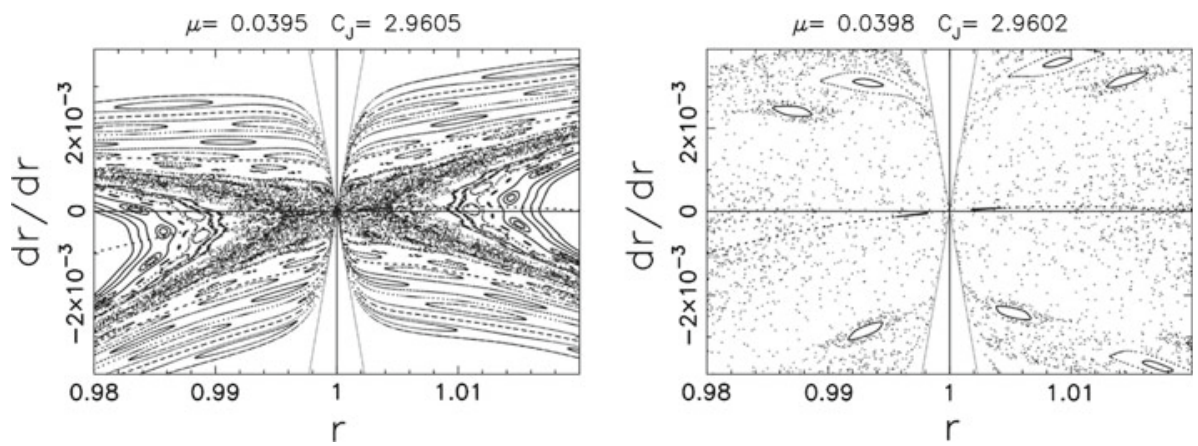

Fig. 3 Left panel Same as Fig. 2, but with $\mu=0.0395$. Right panel Same as Fig. 2, but with $\mu=0.0398$

A particle resting at $L_{4}$ will have $r=1$ and $\dot{r}=0$, so that this particular solution will be represented by a fixed point at the intersection of the vertical $r=1$ and horizontal $\dot{r}=0$ lines, as shown in Figs. 1, 2 and 3.

\section{Global stability of orbits emanating from $L_{4}$ or $L_{5}$}

Figure 1 shows a surface of section obtained slightly above Gascheau's value, namely with $\mu=0.039$, with a close up view of $L_{4}$ vicinity shown in Fig. 2, left panel. The value of the Jacobi constant, $C_{J}=3-\mu=2.961$, ensures that a particle passing at $L_{4}$ is motionless at that point, as explained before. The orbit followed by such a particle is shown in red in the surfaces of section of Figs. 1 and 2. The corresponding orbit in the corotating frame is shown in Fig. 5 [orbit (a)], where it appears to densely fill a limited region around $L_{4}$ in physical space.

The surface of section for $\mu=0.039$ actually indicates that all the orbits surrounding the red one (including the latter) are regular. Thus, globally stable orbits exist around $L_{4}$ for $\mu \geq \mu_{G}$, even if $L_{4}$ is linearly unstable.

One may wonder whether this situation perdures as $\mu$ increases. For $\mu=0.0394$, one can see that the orbit emanating from $L_{4}$ becomes chaotic (Fig. 2, right panel) and can diffuse in phase space. However, because it is surrounded by regular orbits, it cannot escape from $L_{4}$ vicinity. For $\mu=0.0395$ (Fig. 3, left panel), we can observe that the chaotic zone near 


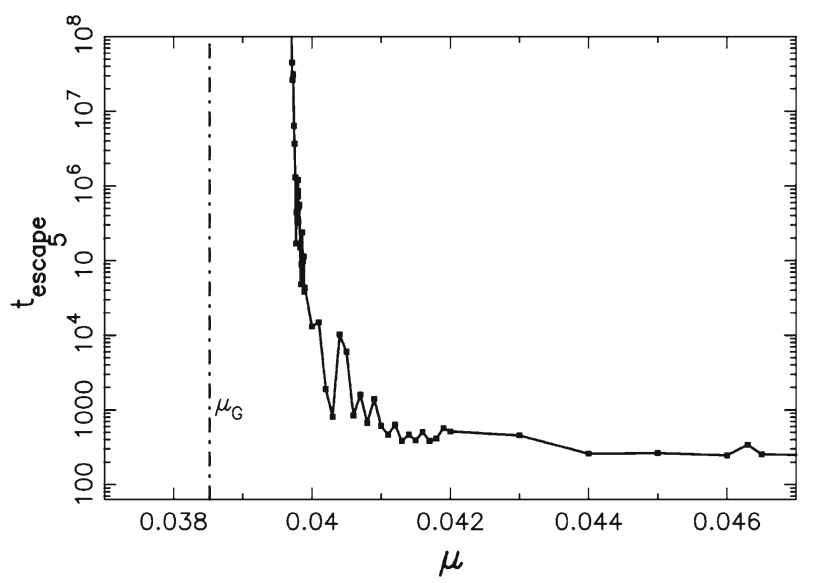

Fig. 4 Escape time of a particle starting motionless at $L_{4}$ (or $L_{5}$ ), as a function of the secondary mass $\mu$, illustrating the global stability of the triangular Lagrangian points. Below $\mu=0.0397$, the particle remains in the $L_{4}$ vicinity over the whole integration time, i.e. until $t=10^{8}$ (i.e. $10^{8} / 2 \pi=1.6 \times 10^{7}$ orbital periods of the secondary). Above $\mu=0.04$, the particle escapes the $L_{4}$ or $L_{5}$ vicinity in less than $t \approx 1000$. Gascheau's value $\mu_{G}$ is shown as the vertical dot-dashed line

$L_{4}(r=1, \dot{r}=0)$ is more and more prevalent, even though regular orbits still prevent escape from $L_{4}$ vicinity.

An abrupt transition occurs just below $\mu=0.04$ : chaos now prevails, the regular orbits fade away (Fig. 3, right panel), and chaotic diffusion allows the particle to escape $L_{4}$ vicinity. Figure 4 shows the survival time $t_{\text {escape }}$ of a particle initially launched motionless at $L_{4}$, i.e. the time after which the particle escapes from the $L_{4}$ vicinity. In all the cases we have examined, this ends up by a close encounter with the secondary, and an escape on a hyperbolic orbit, see an example in the lower part of Fig. 5, with $\mu=0.0405$.

Because the particle has to chaotically diffuse away from $L_{4}$ before escaping, this time of escape is exponentially sensitive to round-off errors and errors associated with the finite order of our integrator, so that it can only be evaluated in a statistical way. Figure 4 actually shows fluctuations of $t_{\text {escape }}$ as $\mu$ increases, as expected in a chaos-dominated process.

The interesting point is that for $\mu \leq 0.0397$, the particle remains in $L_{4}$ vicinity for times larger than the duration of our integration, $t=10^{8}$, while this time drastically drops to less than $10^{3}$ (corresponding to $10^{3} / 2 \pi \sim$ a few hundreds of orbital periods of the primaries) between $\mu=0.0397$ and $\mu \approx 0.04$.

In that sense, the value $\mu \approx 0.04$ may be viewed as a more meaningful stability threshold than $\mu_{G}$, from a physical standpoint. For instance, a spacecraft initially posted still at one of the triangular Lagrange points of a system where $\mu_{G} \leq \mu \leq \approx 0.0397$ will remain around the vicinity of the corresponding triangular point for an infinite, or very long, time, even though the triangular point is linearly unstable.

Previous works actually focussed on the global stability of a particle in the neighborhood of $L_{4}$, for $\mu$ just above $\mu_{G}$. For instance Bardin (2002) shows that a particle sufficienlty close to $L_{4}$ can stay forever in a finite domain around the triangular point for values of $\mu$ larger but sufficienlty close to $\mu_{G}$. Other works investigated the global stability of $L_{4}$ in the frame of the elliptic restricted three-body problem, see Nauenberg (2002) or Érdi et al. (2009). The latter authors have calculated the escape time of a test particle initially motionless at $L_{4}$, in the $\mu-e$ plane, where $e$ is the orbital eccentricity of the primary and secondary. By examining 


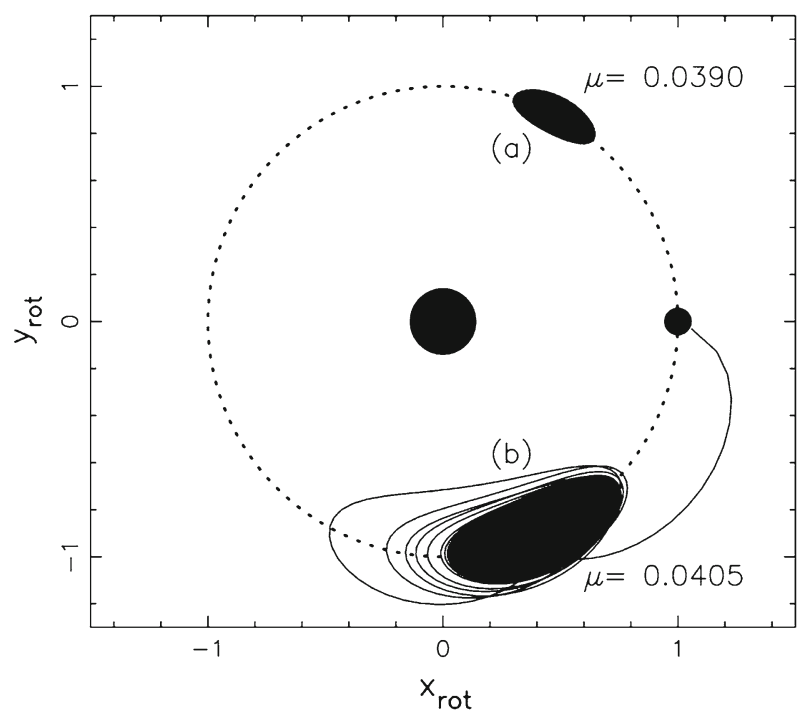

Fig. 5 Two examples of a a globally stable orbit of a particle starting motionless at $L_{4}$ for $\mu=0.039$ (corresponding to the orbit plotted in red in Fig. 1), and $\mathbf{b}$ an unstable orbit starting at $L_{5}$ for $\mu=0.0405$. After a chaotic diffusion inside a region surrounding $L_{5}$, the particle escapes that region at $t \approx 5960$, has a close approach with the secondary, and acquires a hyperbolic orbit. The particle motion is plotted in a frame centered on the primary [larger dot at $\left.\left(x_{\text {rot }}, y_{\text {rot }}\right)=(0,0)\right]$ and corotating with the secondary [smaller dot at $\left.\left(x_{\text {rot }}, y_{\text {rot }}\right)=(1,0)\right]$. The dotted circle centered on the primary has a radius equal to unity

the Fig. 2 of Érdi et al. (2009), one can see that the global stability of $L_{4}$ is actually ensured beyond $\mu=0.03852 \ldots$ when $e=0$. A sharp transition actually occurs around $\mu \approx 0.040$, in agreement with our Fig. 4.

We note finally that particular stable orbits may exist around $L_{4}$ or $L_{5}$ for $\mu \geq 0.04$ (see e.g. Henrard 2002), or even that orbits may connect the two triangular points for values of $\mu$ as large as 0.5 (Henrard and Navarro 2004). So, our present result deals with the global stability of orbits starting at $L_{4}$ or $L_{5}$ for values of $\mu$ just above $\mu_{G}$, and has a statistical meaning only. In particular, it does not exclude the existence of stable orbits for large values of $\mu$.

\section{Periodic orbits around $L_{4}$ or $L_{5}$}

The story of the Lagrange triangular points does not end at $\mu \approx 0.04$, however. Figure 1 shows the presence of two fixed points, labelled "PO", which actually correspond to a unique stable periodic orbit encircling $L_{4}$. This orbit is shown in the upper left corner of Fig. 6 . It intersects the surface of section twice, once for $\dot{\Phi} \geq 0$ (left dot in Fig. 1), and once with $\dot{\Phi} \leq 0$ (right dot in Fig. 1), as explained earlier.

Let us consider a situation where $\mu$ is a parameter slowly varying with time $t$. Then the particle orbit will remain arbitrarily close to a periodic orbit by virtue of the adiabatic invariance of action. This allows us to determine the locus of the fixed points in the $(r, \dot{r})$ phase space, corresponding to various $\mu$ 's. This locus is shown as the dotted curves in Figs. 1, 2 and 3 , and as the solid curves in Fig. 7. 

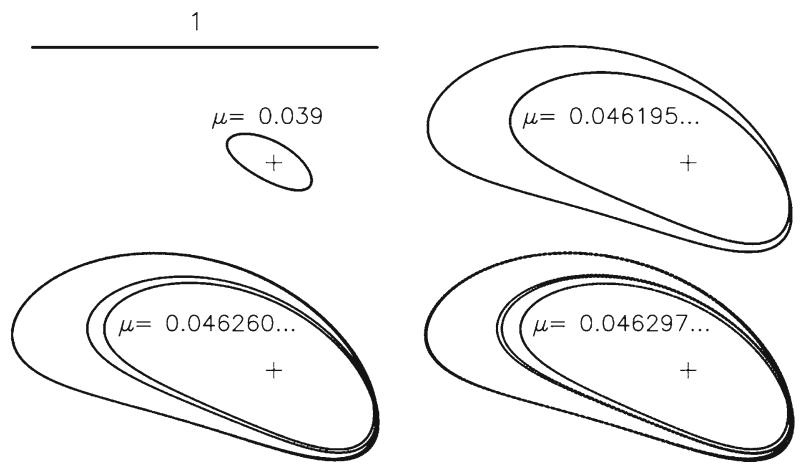

Fig. 6 Examples of four stable periodic orbits beyond Gascheau's value. The crosses indicate the location of the $L_{4}$ point. Each orbit has been displaced with respect to each other for better viewing. The horizontal bar provides the scale. Upper left the periodic orbit corresponding to the fixed points "PO" of Fig. 1, with $\mu=0.039$. Upper right periodic orbit with $\mu=0.0461953059$. This orbit has undergone a period doubling and has now two cycles before completing a period. Lower left periodic orbit corresponding to $\mu=0.0462600179$, with four cycles. Lower right periodic orbit corresponding to $\mu=0.0462979898$, with eight cycles (not all visible at that scale)
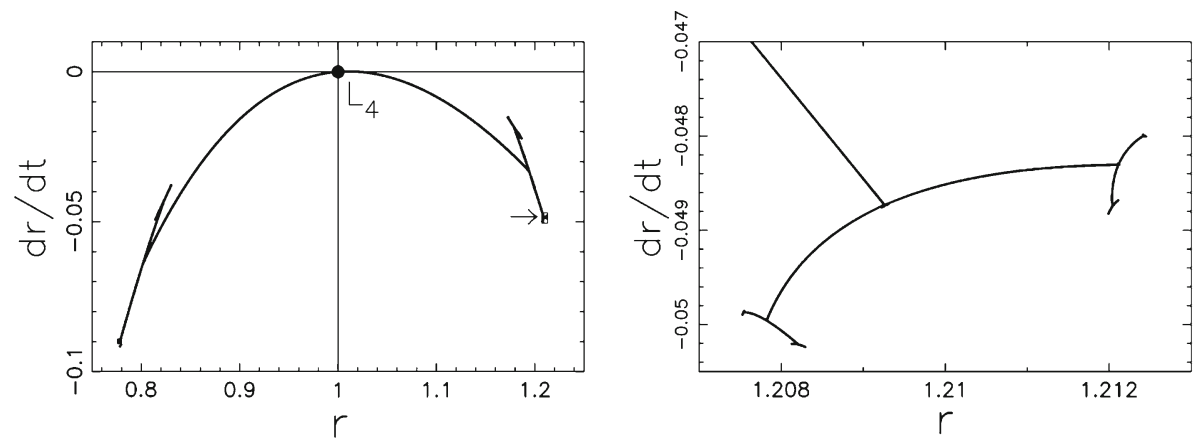

Fig. 7 Left panel locus of the fixed points corresponding to the periodic orbits obtained by slowly varying $\mu$ from Gascheau's value $\mu_{G}$ [at $L_{4}$, i.e. $\left.(r, \dot{r})=(1,0)\right]$ to the limiting value $\mu_{\infty}=0.0463004 \ldots$ (corresponding to the extremities of the arborescence) discussed in the text. Right panel Enlargement of the small box (see arrow) drawn in the left panel

Care must be taken to have a sufficiently slow variation of $\mu$, by choosing a small enough parameter $\epsilon=\mu \dot{(t)} / \mu(t)$. This ensures that the instantaneous orbit for a given $\mu(t)$ stays close enough to the periodic orbit that would be obtained for that particular value of $\mu$. Typical values of $\epsilon$ are $10^{-6}$ near Gascheau's value, and $2 \times 10^{-10}$ near the limiting value $\mu=0.0463004 \ldots$ that will be discussed later.

Figure 6 shows sample examples of periodic orbits obtained as $\mu$ increases. Just above Gascheau's value, the size of the periodic orbit encircling $L_{4}$ rapidly increases while keeping one "braid" (i.e. consisting in a 1-cycle periodic orbit around $L_{4}$ ), then it suffers a period doubling at $\mu=0.045452 \ldots$ and exhibits two braids (i.e. a 2-cycle periodic orbit). A series of period doubling occurs subsequently, with the number of cycles also doubling at each etap, while the size of the orbit keeps on increasing.

Those bifurcations are better seen in the $(r, \dot{r})$ phase space (Fig. 7). One can note the two branches originating from the $L_{4}$ point, and corresponding to 1-cycle periodic orbits. Several ramifications can be observed as $\mu$ increases. An exploration of the phase space around those 

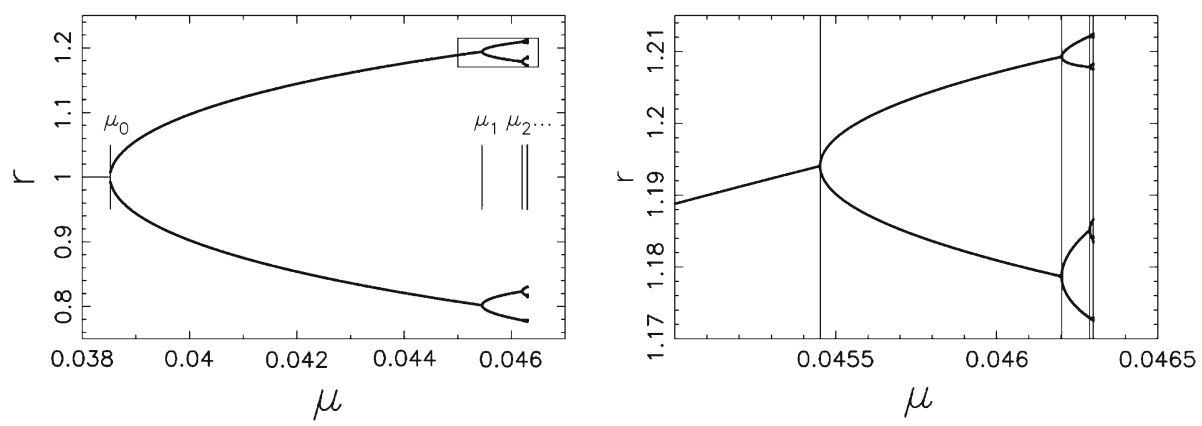

Fig. 8 Left panel Values of $r$ obtained in Fig. 7 as a function of $\mu$. The vertical lines mark the values of $\mu_{i}$ where successive bifurcations occur (see also Table 1). Right panel Enlargement of the box drawn in the left panel

Table 1 Values of the mass ratio $\mu$ at bifurcations

\begin{tabular}{lll}
\hline$i$ & $\mu_{i}$ & $\delta_{i}=\frac{\mu_{i}-\mu_{i-1}}{\mu_{i+1}-\mu_{i}}$ \\
\hline 0 & $0.038520 \ldots$ & - \\
1 & $0.045452 \ldots$ & $9.24 \ldots$ \\
2 & $0.046202 \ldots$ & $8.61 \ldots$ \\
3 & $0.0462891 \ldots$ & $8.66 .$. \\
4 & $0.04629915 \ldots$ & $8.85 \ldots$ \\
5 & $0.046300285 \ldots$ & - \\
\hline
\end{tabular}

fixed points shows that the latters are imprisoned in smaller and smaller regular islands, and surrounded by a more and more prevalent chaotic "sea".

This is the classical behavior of a periodic dynamical system en route to a chaotic behavior, as can be seen in Fig. 8. In that diagram, the values of $r$ obtained in Fig. 7 is now plotted against $\mu$. One recognizes a Feigenbaum cascade, first observed in one-dimensional iterative maps (Feigenbaum 1979). During this cascade, the fixed points of the map suffer period doublings at particular values $\mu_{0}, \mu_{1}, \ldots, \mu_{i}, \ldots$ of $\mu$, each value corresponding to the appearance of a new $2^{i}$-cycle orbit.

It has been shown that the $\mu_{i}$ 's approach geometrically a limiting value $\mu_{\infty}$ as $i \rightarrow \infty$. Actually, there exists a constant $\delta=\lim _{i \rightarrow \infty}\left(\mu_{i}-\mu_{i-1}\right) /\left(\mu_{i+1}-\mu_{i}\right)$, called the Feigenbaum constant, which is common to a wide class of one-dimensional maps (Tabor 1989), with a value of $\delta=4.66920160$. . For area-preserving two-dimensional maps, as it is the case for the Poincaré surface of section examined here, it has been shown that $\delta=8.7210978 \ldots$ (Tabor 1989). Table 1 shows that the ratio $\left(\mu_{i}-\mu_{i-1}\right) /\left(\mu_{i+1}-\mu_{i}\right)$ does get close to that constant as $n$ increases.

\section{Conclusions}

We have examined the dynamics of a particle near the triangular Lagrange's points $L_{4}$ and $L_{5}$, in the frame of the restricted planar and circular three-body problem, and for values of the secondary mass $\mu$ just beyond Gascheau's value $\left.\mu_{\mathrm{G}}=(1-\sqrt{23 / 27}) / 2\right)=0.0385208965 \ldots$, for which $L_{4}$ and $L_{5}$ are linearly unstable. 
We have specifically examined two aspects of the problem. One aspect concerns the global stability of the triangular points, namely the fate of a particle initially placed motionless (in the corotating frame) at $L_{4}$ or $L_{5}$ for $\mu \geq \mu_{G}$. The other aspect concerns the existence of periodic stable orbits encircling $L_{4}$ or $L_{5}$, parametrized by $\mu$, and emanating from the point-like solution at the triangular points for $\mu=\mu_{G}$.

Global stability of the triangular Lagrange points. Figures 2 and 3 indicate that for $\mu \geq$ $\mu_{G}$, the orbit starting at $L_{4}$ or $L_{5}$ first remains regular in a finite interval beyond $\mu_{G}$, e.g. for $\mu=0.039$. In that case, the particle follows a regular orbit that densely fills a finite region near $L_{4}$ or $L_{5}$ [orbit (a) in Fig. 5], although those points are linearly unstable. For larger values of $\mu$ (e.g. 0.0394), an orbit starting at the triangular points is chaotic, but remains nevertheless trapped inside regular orbits in phase space. This ensures that $L_{4}$ and $L_{5}$ are effectively globally stable in the sense that a particle released motionless at $L_{4}$ or $L_{5}$ will remain indefinitely trapped around those points.

Between $\mu=0.0397$ and $\mu=0.04$, the regular "imprisoning orbits" disappear, and chaotic diffusion allows the particle to escape from $L_{4}$ or $L_{5}$ vicinity [orbit (b) in Fig. 5]. Thus, the value $\mu \approx 0.04$ should be regarded as the threshold beyond which the triangular Lagrange points $L_{4}$ and $L_{5}$ are no longer globally stable.

Stable periodic orbits around the triangular Lagrange points. There exists a family of stable periodic orbits encircling $L_{4}$ or $L_{5}$, parametrized by $\mu$ beyond Gascheau's value. Those orbits start as a point at $L_{4}$ or $L_{5}$ for $\mu=\mu_{G}$, and then increase in size as $\mu$ increases (see upper left panel of Fig. 6).

This family undergoes a bifurcation at $\mu_{1}=0.045452 \ldots$ with the appearance of two cycles in the orbit, yielding a period doubling (upper right panel of Fig. 6). A series of bifurcations is subsequently observed at values $\mu_{i}$, each yielding new $2^{i}$-cycle orbits (Table 1 ).

Those bifurcations are readily observed in the phase space $(r, \dot{r})$, see Fig. 7 , and in a plot of $r$ versus $\mu$ (Fig. 8). We recognize a classical Feigenbaum cascade, with a series of period doublings, eventually yielding to chaos. The series $\mu_{i}$ closely follows a geometrical progression, with an associated Feigenbaum constant $\delta=\lim _{n \rightarrow \infty}\left(\mu_{i}-\mu_{i-1}\right) /\left(\mu_{i+1}-\right.$ $\mu_{i}$ ) consistent with the value expected from area-preserving two-dimensional maps, $\delta=$ $8.7210978 \ldots$

A closer examination of the branchings in Fig. 8, and an extrapolation of the series using the Feigenbaum constant both provide a limiting value of $\mu_{\infty}=0.0463004 \ldots$ for the mass ratio at which the periodic orbits surrounding $L_{4}$ or $L_{5}$ dissolve themselves into a chaotic region of the phase space. So, it appears that Gascheau's value $\mu_{G}$ is the first of a series $\mu_{0}\left(=\mu_{G}\right), \mu_{1}, \ldots, \mu_{i}, \ldots$ corresponding to successive period doublings, and leading to the disappearance of this family of periodic orbits for $\mu_{\infty}=0.0463004 \ldots$. The latter quantity may be thus considered as a limiting value which generalizes the finding of Gascheau.

As a final remark, we note that in Feigenbaum's diagrams, there may exist small intervals of $\mu$ beyond $\mu_{\infty}$ where regular behavior shortly appears, although chaos prevails in those regions. So, we do not exclude the existence of stable periodic orbits around $L_{4}$ and $L_{5}$ beyond $\mu_{\infty}=0.0463004 \ldots$ Exploring this possibility remains, however, out of the scope of this paper.

Acknowledgments I would like to thank Jacques Henrard, who substantially contributed to this paper with detailed discussions and by sending me several reprints on the dynamics of the Lagrange points. I also thank Michel Hénon for providing reprints on that topic. I furthermore benefited from discussions and insights from Alain Albouy and Giancarlo Benettin. We acknowledge the reviewers for constructive comments. 


\section{References}

Bardin, B.: On motions near the Lagrange equilibrium point $L_{4}$ in the case of Routh's critical mass ratio. Celest. Mech. Dyn. Astron. 82, 163 (2002)

Bennett, A.: Characteristic exponents of the five equilibrium solutions in the elliptical restricted problem. Icarus 4, 177 (1965)

Danby, J.M.A.: Stability of the triangular points in the elliptic restricted problem of three bodies. Astron. J. 69, 165 (1964)

Deprit, A., Deprit-Bartholomé, A.: Stability of the triangular Lagrangian points. Astron. J. 72, 173 (1967)

Efthymiopoulos, C.: Formal integrals and Nekhoroshev stability in a mapping model for the Trojan asteroids. Celest. Mech. Dyn. Astron. 92, 29 (2005)

Érdi, B., Forgács-Dajka, E., Nagy, I., Rajnai, R.: A parametric study of stability and resonances around $L_{4}$ in the elliptic restricted three-body problem. Celest. Mech. Dyn. Astron. 104, 145 (2009)

Euler, L.: Considérations sur le problème des trois corps. Mém. de Lacad. d. Sc. de Berlin 19, 194 (1763)

Euler, L.: Considerationes de motu corporum coelestium. Novi Commentarii Academiae Scientiarum Petropolitanae 10, 544 (1764)

Euler, L.: De motu rectilineo trium corporum se mutuo attrahentium. Novi Commentarii Academiae Scientiarum Petropolitanae 11, 144 (1765)

Feigenbaum, M.J.: The universal metric properties of nonlinear transformations. J. Stat. Phys. 21, 669 (1979)

Gascheau, G.: Examen d'une classe d'équations différentielles et applications à un cas particulier du problème des trois corps. C. R. Acad. Sci. 16, 393 (1843)

Henrard, J.: The web of periodic orbits at $L_{4}$. Celest. Mech. Dyn. Astron. 83, 291 (2002)

Henrard, J., Navarro, J.F.: Families of periodic orbits emanating from homoclinic in the restricted problem of three bodies. Celest. Mech. Dyn. Astron. 89, 285 (2004)

Lagrange, J.L.: Essai sur le problème des trois corps, 229. In: Serret, J.-L. (ed.) Euvres de Lagrange, Tome sixième, Gauthier-Villard, Paris (1873)

Marchal, C.: The three-body problem, pp. 46-51. Elsevier, Amsterdam (1990)

Murray, C.D., Dermott, S.F.: Solar System Dynamics, 93. Cambridge University Press, Cambridge (1999)

Nauenberg, M.: Stability and eccentricity for two planets in a 1:1 resonance, and their possible occurrence in extrasolar planetary systems. Astron. J. 124, 2332 (2002)

Routh, E.J.: On Laplace's three particles, with a supplement on the stability of steady motion. Proc. London Math. Soc. 6, 86 (1875) (repr. in Stability of Motion, Fuller, A.T. (ed.) [London: Taylor \& Francis], Chap. 3 [1975])

Routh, E.J.: Dynamics of a System of Rigid Bodies, part. II, p. 76, 6th edn. Macmillan, London (1905) (reprint Dover Publication, New York, 1955)

Santoprete, M.: Linear stability of the Lagrangian triangle solutions for quasihomogeneous potentials. Celest. Mech. Dyn. Astron. 94, 17 (2006)

Sándor, Z., Érdi, B., Efthymiopoulos, C.: The phase space structure around L4 in the restricted three-body problem. Celest. Mech. Dyn. Astron. 78, 113 (2000)

Tabor, M.: Chaos and Integrability in Nonlinear Dynamics: An Introduction, pp. 220-225. Wiley, New York (1989) 